\title{
Processing of Emotional Reactivity and Emotional Memory over Sleep
}

\author{
Bengi Baran, ${ }^{1}$ Edward F. Pace-Schott, ${ }^{1,2}$ Callie Ericson, ${ }^{1}$ and Rebecca M. C. Spencer ${ }^{1,2}$ \\ ${ }^{1}$ Department of Psychology and ${ }^{2}$ Neuroscience and Behavior Program, University of Massachusetts, Amherst, Amherst, Massachusetts 01003
}

Sleep enhances memories, particularly emotional memories. As such, it has been suggested that sleep deprivation may reduce posttraumatic stress disorder. This presumes that emotional memory consolidation is paralleled by a reduction in emotional reactivity, an association that has not yet been examined. In the present experiment, we used an incidental memory task in humans and obtained valence and arousal ratings during two sessions separated either by $12 \mathrm{~h}$ of daytime wake or $12 \mathrm{~h}$ including overnight sleep. Recognition accuracy was greater following sleep relative to wake for both negative and neutral pictures. While emotional reactivity to negative pictures was greatly reduced over wake, the negative emotional response was relatively preserved over sleep. Moreover, protection of emotional reactivity was associated with greater time in REM sleep. Recognition accuracy, however, was not associated with REM. Thus, we provide the first evidence that sleep enhances emotional memory while preserving emotional reactivity.

\section{Introduction}

Accumulating evidence points to an enhancing effect of sleep on memory consolidation (Stickgold, 2005). Performance on a range of tasks is better after an interval of sleep compared with an equivalent interval spent awake even after controlling for possible circadian influences on performance (Walker and Stickgold, 2006; Marshall and Born, 2007; Diekelmann and Born, 2010). Studies that reveal direct relationships between physiological properties of sleep and changes in task performance over sleep suggest that sleep actively modulates memory representations (Gais and Born, 2004; Schabus et al., 2004; Marshall et al., 2006).

Emotional but not neutral memories show enhanced consolidation over a full night of sleep compared with an equivalent interval of wake (Hu et al., 2006; Payne et al., 2008). Likewise, Nishida et al. (2009) showed that participants who napped, compared with those who remained awake, showed preferential enhancement of memory for negative but not neutral pictures, the extent of which was correlated with the amount of REM in the nap. Other studies have illustrated the preferential consolidation of memory for emotional narratives following sleep relative to wake. For instance, Wagner et al. (2001) found that retrieval of emotionally negative, but not neutral, narrative texts was better after REM-rich late-night sleep compared with slow-wave sleep (SWS)-rich early night sleep or an equivalent interval awake, an effect persisting up to 4 years later (Wagner et al., 2006).

It has also been proposed that sleep modulates emotional reactivity, a construct that can be described along two orthogonal

Received May 20, 2011; revised Nov. 28, 2011; accepted Dec. 1, 2011.

Author contributions: B.B., E.F.P.-S., and R.M.C.S. designed research; B.B. and C.E. performed research; B.B.,

E.F.P.-S., C.E., and R.M.C.S. analyzed data; B.B., E.F.P.-S., and R.M.C.S. wrote the paper.

This study was supported by NIH Grant R00 AG029710.

The authors have no financial conflicts of interest.

Correspondence should be addressed to Dr. Rebecca M. C. Spencer, Department of Psychology, 419 Tobin Hall,

135 Hicks Way Amherst, MA 01003. E-mail: rspencer@psych.umass.edu.

DOI:10.1523/JNEUROSCI.2532-11.2012

Copyright $\odot 2012$ the authors $\quad 0270-6474 / 12 / 321035-08 \$ 15.00 / 0$ dimensions of valence (sad to happy) and arousal (calm to excited) (LaBar and Cabeza, 2006; Bradley and Lang, 2007). The high incidence of sleep abnormalities in mood disorders (Riemann, 2007) may suggest that sleep interacts with emotional processing. Moreover, emotionally guided decision making is enhanced by sleep (Pace-Schott et al., 2011b) and impaired by sleep deprivation (Killgore et al., 2006). Sleep also modulates low-level emotional processes such as extinction and habituation (PaceSchott et al., 2009, 2011a).

Such lines of evidence have been used to support the hypothesis that sleep deprivation following trauma may serve as an intervention to prevent PTSD (Wagner et al., 2006). Accordingly, as sleep enhances consolidation of the emotional memory, the emotional reactivity will also be maintained and, conversely, by restricting sleep following trauma, memories will fail to be consolidated and reactivity will be reduced. An alternative model by Walker (Walker, 2009; Walker and van der Helm, 2009) suggests that when emotional memories are consolidated over sleep, negative emotional tone is simultaneously attenuated.

Underlying these hypotheses is the assumption that sleepdependent memory consolidation and sleep-dependent changes in emotional reactivity are comodulated. However, the relationship between changes in emotional reactivity and memory processing over sleep has not yet been examined. Wagner and colleagues (2002) reported that subjective ratings of valence following sleep are more negative for pictures previewed before sleep relative to unfamiliar pictures, a change associated with time spent in REM. However, memory for the items following sleep and wake was not assessed. The goal of the present study was to examine whether emotional memory and reactivity are comodulated. Moreover, using polysomnography, we investigated whether REM sleep played a particular role in emotion processing.

\section{Materials and Methods}

Participants. Participants were 106 young adults (68 female, 38 male) between 18 and 30 years (mean $=20.4$ years, $S D=2.2$ ) with no history 
Table 1. Valence and arousal ratings

\begin{tabular}{|c|c|c|c|c|}
\hline & \multicolumn{2}{|l|}{ Valence } & \multicolumn{2}{|l|}{ Arousal } \\
\hline & Targets & Foils & Targets & Foils \\
\hline \multicolumn{5}{|l|}{ Negative } \\
\hline \multicolumn{5}{|l|}{ Norms } \\
\hline Range & $1.51-3.25$ & $1.52-3.42$ & $3.18-7.64$ & $2.34-6.53$ \\
\hline Mean (SEM) & $2.28(0.12)$ & $2.67(0.12)$ & $5.56(0.37)$ & $4.48(0.31)$ \\
\hline \multicolumn{5}{|c|}{ Session 1, Mean (SEM) } \\
\hline Sleep & $1.72(0.06)$ & & $6.32(0.22)$ & \\
\hline Wake & $1.77(0.09)$ & & $5.54(0.38)$ & \\
\hline Evening & $1.72(0.13)$ & & $7.05(0.18)$ & \\
\hline Morning & $1.64(0.11)$ & & $6.56(0.36)$ & \\
\hline \multicolumn{5}{|c|}{ Session 2, Mean (SEM) } \\
\hline Sleep & $2.11(0.09)$ & $1.89(0.07)$ & $5.72(0.23)$ & $5.93(0.27)$ \\
\hline Wake & $2.41(0.12)$ & $2.14(0.09)$ & $4.37(0.34)$ & $4.91(0.46)$ \\
\hline Evening & $2.18(0.15)$ & $2.03(0.11)$ & $6.98(0.24)$ & $6.81(0.25)$ \\
\hline Morning & $2.29(0.14)$ & $1.92(0.15)$ & $6.40(0.33)$ & $5.93(0.52)$ \\
\hline \multicolumn{5}{|l|}{ Neutral } \\
\hline \multicolumn{5}{|l|}{ Norms } \\
\hline Range & $4.99-5.99$ & $4.90-5.64$ & $1.19-4.25$ & $1.17-4.27$ \\
\hline Mean (SEM) & $5.48(0.09)$ & $5.27(0.07)$ & $2.23(0.26)$ & $2.32(0.26)$ \\
\hline \multicolumn{5}{|c|}{ Session 1, Mean (SEM) } \\
\hline Sleep & $5.02(0.02)$ & & $2.28(0.15)$ & \\
\hline Wake & $5.04(0.02)$ & & $2.59(0.27)$ & \\
\hline Evening & $5.32(0.09)$ & & & \\
\hline Morning & $5.36(0.11)$ & & & \\
\hline \multicolumn{5}{|c|}{ Session 2, Mean (SEM) } \\
\hline Sleep & $4.88(0.04)$ & $4.91(0.02)$ & $2.15(0.12)$ & $2.12(0.15)$ \\
\hline Wake & $4.90(0.06)$ & $4.93(0.04)$ & $2.36(0.23)$ & $2.52(0.30)$ \\
\hline Evening & $5.29(0.09)$ & $4.91(0.03)$ & $2.33(0.34)$ & $2.42(0.29)$ \\
\hline Morning & $4.96(0.12)$ & $4.90(0.04)$ & $2.14(0.36)$ & $2.32(0.36)$ \\
\hline
\end{tabular}

of neurological or sleep disorder, head injury, and no current use of medications known to affect cognitive functioning or sleep. Participants were excluded for habitual overnight sleep of $<5 \mathrm{~h}$. All participants were native English speakers with normal or corrected-to-normal vision. Testing procedures were approved by the University of Massachusetts, Amherst Institutional Review Board and written informed consent was obtained before the experiment. Participants were compensated with course credit or payment.

Materials. Stimuli were 180 pictures (90 negative, 90 neutral). Of these, 110 of the pictures (55 in each valence category) were taken from the International Affective Picture System (IAPS) (Lang et al., 2005); the rest of the images came from an in-house picture set, which had images that were categorically similar to the IAPS set (e.g., objects, scenes, animals). To balance valence and arousal in two sets (targets and foils) a priori, all stimuli (IAPS and in-house) were rated by 15 participants in a preliminary study. Target and foil picture sets were similar in Valence $\left(t_{(14)}=-1.11, p=0.28\right)$ and Arousal $\left(t_{(14)}=\right.$ $1.55, p=0.14$ ) dimensions (Table 1 ).

Procedure. The experiment consisted of two phases (Fig. 1). In the Encoding Phase, each picture was presented on a computer screen for $1000 \mathrm{~ms}$ with an interstimulus interval of $1500 \mathrm{~ms}$. Participants viewed 60 pictures (30 negative and 30 neutral). After each picture, a nine-item self-assessment manikin (SAM) valence scale (Lang, 1980) was displayed and participants were prompted to rate the valence of the preceding picture using the numbers on the keyboard ( $1=\operatorname{sad}, 9=$ happy). This was followed by presentation of a nine-item SAM arousal scale $(1=\mathrm{calm}$, $9=$ excited), prompting participants to rate the subjective arousal of the preceding picture. There were no time restrictions on responses for valence and arousal ratings.

Subsequently, participants completed a surprise Recognition Phase. As such, this was an incidental memory task. In the Recognition Phase, stimuli were the pictures from the Encoding Phase (targets) intermingled with 120 novel pictures (60 negative, 60 neutral; foils) presented for 1000 ms. Participants again rated pictures for valence and arousal followed by a yes/no memory judgment (i.e., "Have you seen this picture before?"). Responses were made at the participant's own pace.
Participants were assigned to one of four groups. The Sleep group (18 male, 36 female) started their first session in the evening (beginning between 8:00 and 10:00 P.M.) and the second session was $12 \mathrm{~h}$ later following a full night's sleep. The Wake group (14 male, 14 female) started their first session in the morning (beginning between 8:00 and 10:00 A.M.) and the second session took place $12 \mathrm{~h}$ later following continuous waking. Participants were instructed not to nap or consume alcohol between sessions. At the end of each session, all participants filled out a sleep diary and at the end of their second session they completed the Pittsburgh Sleep Quality Index (PSQI) (Buysse et al., 1989), which surveys habitual sleep quality estimates from the preceding $30 \mathrm{~d}$.

The Morning and Evening groups were included to rule out the possibility that memory performance or emotionality ratings varied due to circadian effects on cognition (e.g., attention). The Evening group (4 male, 8 female) was tested between 8:00 and 10:00 P.M. and the Morning group ( 2 male, 10 female) was tested between 8:00 and 10:00 A.M. A 45 min break between the Encoding and Recognition Phases was spent in the lab at which time participants filled out questionnaires and watched a nature video in any remaining time.

Polysomnography. Polysomnography (PSG) was recorded for 25 participants in the Sleep group with the Aura PSG ambulatory system (Grass Technologies). The PSG montage was applied in the participants' place of living $\sim 1 \mathrm{~h}$ before the participant's estimated bedtime. The montage included EOG (right and left ocular canthus), two chin EMG, and four cortical EEG leads (O1, O2, C3, C4) with all electrodes referenced to Cz. Data were obtained and analyzed according to the specifications provided in the revised AASM manual (Iber et al., 2007).

Statistical analyses. Initial subjective ratings of valence were used to categorize stimuli for subsequent analyses (St. Jacques et al., 2009). Valence was chosen as the grouping dimension because it is the predominant dimension used in previous studies (Wagner et al., 2001; Payne et al., 2008; Nishida et al., 2009). Targets were separated based on valence ratings during the Encoding Phase (session 1) and foils were classified based on valence ratings in the Recognition Phase (session 2). Those stimuli that participants rated 1-3 created the negative category and pictures rated 4-6 formed the neutral category. Thus, the negative and neutral picture sets were unique for each participant and the exact number of items for each valence category differed among participants. Across participants in the $12 \mathrm{~h}$ groups, there was an average of $24.2(\mathrm{SD}=$ 3.8) negative and $28.9(\mathrm{SD}=5.6)$ neutral target pictures. A mixed ANOVA on the number of targets revealed no significant main effect of Group (Sleep vs Wake; $F_{(1,79)}=.001, p=0.97$ ), a significant effect of Valence with more neutral targets than negative targets $\left(F_{(1,79)}=17.72\right.$, $p<0.001)$ but, importantly, the Group $\times$ Valence interaction was not significant $\left(F_{(1,79)}=.02, p=0.90\right)$. The average number of negative foils was $48.02(\mathrm{SD}=11.12)$ and the average number of neutral foils was 64.24 $(\mathrm{SD}=11.35)$. A mixed ANOVA on the number of foils revealed no significant main effect of Group $\left(F_{(1,79)}=0.23, p=0.64\right)$, an effect of Valence with more neutral foils than negative foils $\left(F_{(1,79)}=34.35, p<\right.$ $0.001)$, but no significant Group $\times$ Valence interaction $\left(F_{(1,79)}=0.01\right.$, $p=0.92$ ).

Given this manner of classifying stimuli by valence, which may give the most accurate valence classification to targets (seen in Sessions 1 and 2) and that our primary measures of emotional reactivity are based on targets, our primary memory measure is Hit Rate. Hit Rate is defined as the percentage of items correctly identified as previously seen. The secondary memory measures were False Alarms (i.e., percentage of items incorrectly identified as previously seen), recognition memory discriminability $\left(d^{\prime}\right)$ and response bias $(c)$.

Change in valence ratings was computed for target pictures (shown at both sessions): $\Delta$ Valence $=$ valence rating for Session $2-$ valence rating for Session 1.

Accordingly, a positive $\Delta$ Valence score for a negative picture corresponded to decrease of the initial negative reaction (toward neutrality). Similarly change in subjective arousal was calculated as $\Delta$ Arousal $=$ arousal rating for Session 2 - arousal rating for Session 1. A negative $\Delta$ Arousal score would mean that the picture was rated as less arousing in the second session. 
A
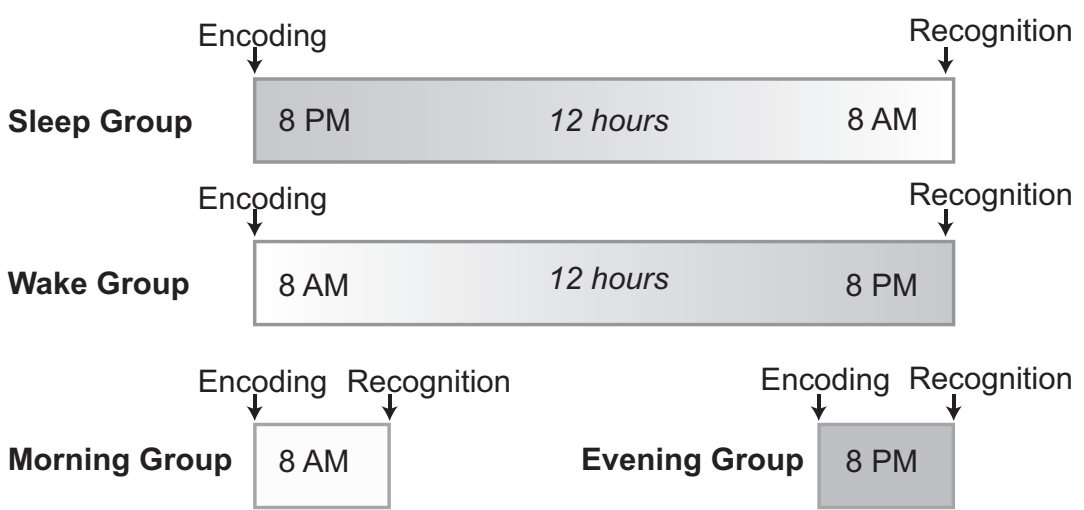

B

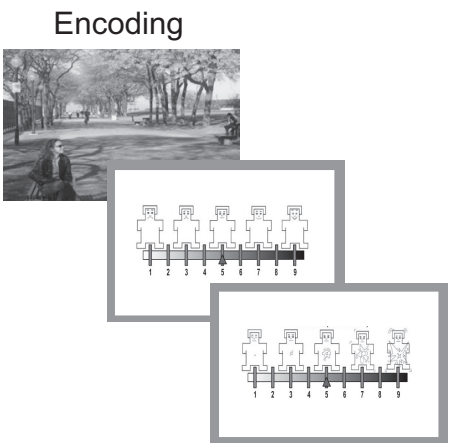

60 targets

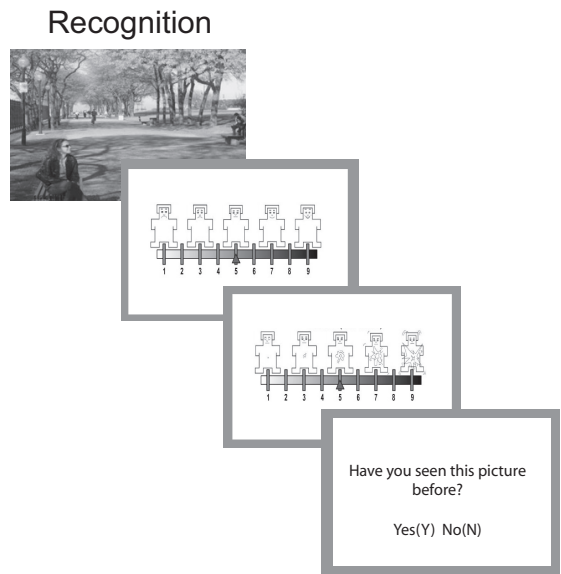

60 targets +120 foils

Figure 1. Study design and memory task. $\boldsymbol{A}$, There were four groups. For the Sleep and Wake groups, the Encoding and Recognition Phases were separated by $12 \mathrm{~h}$. The Morning and Evening control groups performed these phases within a session, $\sim 45$ min apart. $B$, The task was an incidental memory task. During the Encoding Phase, participants viewed 60 pictures and rated the valence and arousal of each picture. During the Recognition Phase, participants viewed 60 target pictures intermingled with 120 foil pictures. Following the presentation of each picture, participants rated valence and arousal and made a yes/no memory judgment.

Memory measures were compared using two-factor mixed ANOVA with a between-subjects factor Group (Sleep vs Wake) and a withinsubjects factor Valence (Negative vs Neutral). Similarly, $\Delta$ Valence and $\Delta$ Arousal were compared using two-factor mixed ANOVA with a between-subjects factor Group (Sleep vs Wake) and a within-subjects factor Valence (Negative vs Neutral). The Greenhouse-Geisser correction was applied to within-subject main effects and their interactions. Analyses of covariance examined Group differences in Negative and Neutral hit rates while taking into account, in separate (single covariate) analyses, global PSQI scores and Gender. One-way ANOVAs compared groups' global PSQI scores, subjective habitual sleep latency and duration estimates, and their self-reported sleep duration and sleep latency on experimental nights.

To more thoroughly investigate relationships between the main dependent variables (Hit Rate, $\Delta$ Valence, and $\Delta$ Arousal) and sleep stages, both correlation matrices (Pearson's $r$ ) and forward stepwise regressions were calculated. Analyses were performed for sleep-stage percentages across the entire night. Additionally, we separately compared performance to measures of REM in the third quarter of the night (REM3\%) and in the fourth quarter of the night (REM4\%). To do so, total sleep time was divided by four and the percentage of the third and fourth quarters spent in REM were calculated. Likewise, given that SWS is predominant in the first half of the night, we separately analyzed SWS in the first (SWS1\%) and second (SWS2\%) quarters of the night.

Possible circadian influences on performance were investigated by comparing Morning and Evening control groups on the primary out- come measures with two-factor mixed ANOVAs. Furthermore, effects of time and wakefulness on emotional reactivity were investigated by comparing $\Delta$ Valence and $\Delta$ Arousal between $12 \mathrm{~h}$ groups (Wake and Sleep) and $45 \mathrm{~min}$ control groups (Evening and Morning) using one-way ANOVAs.

\section{Results}

\section{Sleep characteristics}

Participants were similar in habitual sleep characteristics based on PSQI reports, a subjective measure of sleep quality over the past $30 \mathrm{~d}$ (Sleep group mean PSQI score $=4.8$; Wake group mean PSQI score $\left.=5.4 ; F_{(1,80)}=0.97, p=0.33\right)$. Subjectively estimated habitual sleep latency $\left(F_{(1,80)}=0.16, p=0.69\right)$ and habitual sleep duration $\left(F_{(1,80)}=0.05, p=0.82\right)$ also did not differ for PSQI reports across groups.

Full-night PSG was measured for 25 of the participants in the Sleep group. Total PSQI, habitual sleep latency, and habitual sleep duration did not differ between Sleep groups with and without PSG (all ps > 0.49). Subjective reports of total sleep time for the experimental night did not differ for those Sleep group participants with PSG (mean $=403.5 \mathrm{~min}, \mathrm{SD}=68.1)$ and those without PSG $($ mean $=362.7 \mathrm{~min}, \mathrm{SD}=$ 96.6), although this trended toward significance $\left(F_{(1,52)}=3.12, p=0.08\right)$. Note that, given this trend, behavioral measures were compared between Sleep group participants with and without PSG. Recognition accuracy and valence ratings did not differ between groups with the exception of arousal ratings, which showed a greater decrease in the no PSG group $\left(F_{(1,52)}=5.1, p=0.03\right)$. Sleep latency was $16.8 \mathrm{~min}(\mathrm{SD}=13.4)$ for the Sleep group without PSG, as reported in sleep diaries. This did not differ from subjective sleep latency for the Sleep group with PSG, $22.6 \min \left(F_{(1,52)}=1.81, p=0.19\right)$.

Within the PSG group, a comparison of self-reported sleep time and sleep time as measured by PSG neared significance $\left(t_{(24)}=-1.67, p=0.11\right)$ with participants' subjective reports tending to underestimate total sleep time. Such misperceptions have been observed by others (Carskadon et al., 1976; Silva et al., 2007). PSG measured mean sleep efficiency was 92.84\% (SD = 6.19). Participants spent 23\% (SD = 18.52) of total sleep time in non-REM (NREM) Stage 1 sleep, 35\% $(\mathrm{SD}=15.12)$ in NREM Stage $2,21.78 \%(\mathrm{SD}=6.38)$ in SWS, and $23.67 \%(\mathrm{SD}=9.28)$ in REM sleep.

\section{Hit rate}

Table 2 presents hit rate (HR) and false alarms (FA) across groups and emotional valence categories. An ANOVA on HR revealed that the main effect of group (Sleep vs Wake) was significant $\left(F_{(1,80)}=10.27, p=0.002\right)$. That is, for both negative and neutral pictures, the Sleep group performed better than the Wake group (Fig. 2A). The main effect of Valence was also significant, with negative pictures remembered better than neutral pictures, 
$\left(F_{(1,80)}=38.65, p<0.001\right)$. The interaction between Valence and Group was not significant $\left(F_{(1,80)}=1.15, p=0.29\right)$, suggesting that the benefit of sleep after encoding was not modulated by stimulus valence.

An ANOVA on FA revealed a significant main effect of Group, with Sleep group participants having fewer false alarms than the Wake group $\left(F_{(1,79)}=4.91, p=0.03\right)$. Neither the main effect of Valence $\left(F_{(1,79)}=1.25, p=0.23\right)$ nor the Valence $\times$ Group interaction $\left(F_{(1,79)}=0.69, p=0.41\right)$ was significant.

Individual differences in habitual sleep quality did not confound group comparisons for hit rate. By adding total PSQI scores to the mixed ANOVA model, the Group main effect remained significant $\left(F_{(1,74)}=6.45, p=0.013\right)$ with neither PSQI main effect nor PSQI $\times$ Group interactions. Given that gender was not balanced across groups, Gender was added to the model. The Group main effect remained significant $\left(F_{(1,78)}=12.68, p<\right.$ $0.001)$, but only a trend toward a Gender main effect $\left(F_{(1,74)}=\right.$ $2.96, p=0.09$, Males greater) emerged. Importantly, there was no significant Group $\times$ Gender or Valence $\times$ Group $\times$ Gender interactions (both $p s>0.11$ ).

\section{Recognition memory}

It is possible that the analyses of Hit Rate reflect differences in response bias rather than memory. Based on the signal detection theory (Macmillan and Creelman, 1990), memory discrimination is most accurately summarized by measures of sensitivity (the ability to discriminate hit rates and false alarms; $d^{\prime}$ ) and response bias (tendency for conservative vs liberal responding; $c$ ). An ANOVA on $d^{\prime}$ revealed a significant main effect of Group with better recognition memory discriminability in the Sleep group $\left(F_{(1,79)}=14.63, p<\right.$ 0.001 ), a significant main effect of Valence with better memory for negative pictures $\left(F_{(1,79)}=20.87, p<0.001\right)$, and no significant Group $\times$ Valence interaction $\left(F_{(1,79)}=\right.$ $0.99, p=0.32)$. Response bias, $c$, was similar between Sleep and Wake groups $\left(F_{(1,79)}=\right.$ $0.05, p=0.82)$. There was a main effect of Valence with more conservative response tendency for neutral pictures $\left(F_{(1,79)}=\right.$ 25.32, $p<0.001)$ but no Group $\times$ Valence interaction $\left(F_{(1,79)}=0.01, p=0.93\right)$.

\section{Valence and Arousal}

Session 1 subjective reports of valence and arousal allow us to probe baseline differences between groups, at least with respect to emotional reactivity. Importantly, there were no baseline differences. Sleep and Wake groups rated neutral $\left(F_{(1,79)}=0.20\right.$, $p=0.65)$ and negative $\left(F_{(1,79)}=0.26, p=\right.$ $0.61)$ items similarly for Valence. Likewise, Arousal ratings were similar for Sleep and Wake groups (neutral: $F_{(1,79)}=1.65, p=0.28$; negative: $F_{(1,79)}=$ $3.53, p=0.074)$. Although we note the trend toward significance of the latter comparison, by focusing on delta ( $\Delta$ Arousal), the change in ratings over the break, this possible difference is accounted for. Notably, this analysis also suggests that the subjective ratings did not differ by time of day, discounting circadian influences on this task.
Table 2. Memory accuracy across groups separated by valence

\begin{tabular}{|c|c|c|c|}
\hline & Mean & SEM & $F$ \\
\hline \multicolumn{4}{|l|}{ Hit rate (\%) } \\
\hline \multicolumn{4}{|l|}{ Negative } \\
\hline Sleep & 82.26 & 1.41 & \\
\hline Wake & 76.17 & 2.15 & $5.53^{*}$ \\
\hline Evening & 87.42 & 2.57 & \\
\hline Morning & 81.66 & 2.46 & 3.27 \\
\hline \multicolumn{4}{|l|}{ Neutral } \\
\hline Sleep & 74.00 & 1.85 & \\
\hline Wake & 64.46 & 2.83 & $8.51^{* *}$ \\
\hline Evening & 80.91 & 2.57 & \\
\hline Morning & 77.43 & 3.38 & 0.67 \\
\hline \multicolumn{4}{|l|}{ False alarms (\%) } \\
\hline \multicolumn{4}{|l|}{ Negative } \\
\hline Sleep & 16.47 & 1.41 & \\
\hline Wake & 19.85 & 1.48 & 2.32 \\
\hline Evening & 12.12 & 2.57 & \\
\hline Morning & 11.56 & 2.16 & 0.49 \\
\hline \multicolumn{4}{|l|}{ Neutral } \\
\hline Sleep & 14.70 & 1.09 & \\
\hline Wake & 19.52 & 1.51 & $6.74^{*}$ \\
\hline Evening & 12.41 & 2.16 & \\
\hline Morning & 10.80 & 3.11 & 0.49 \\
\hline
\end{tabular}
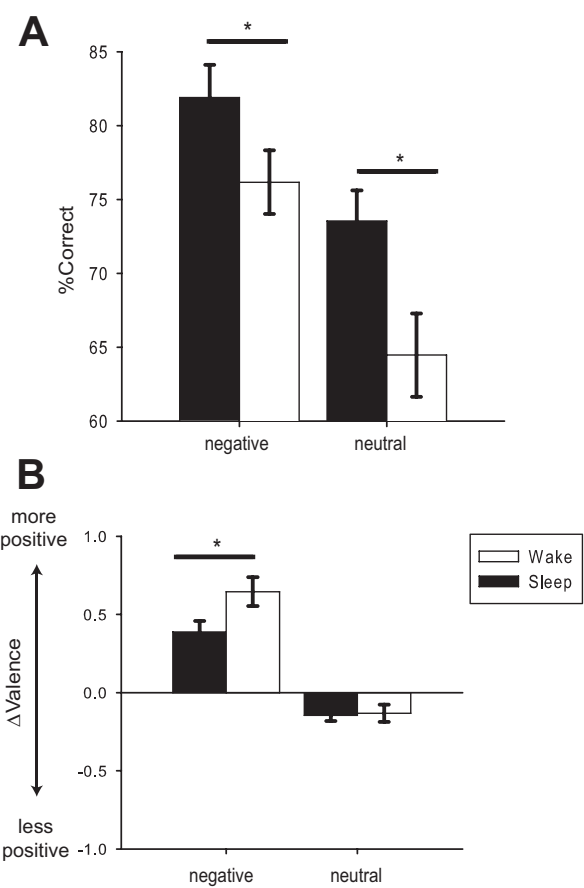

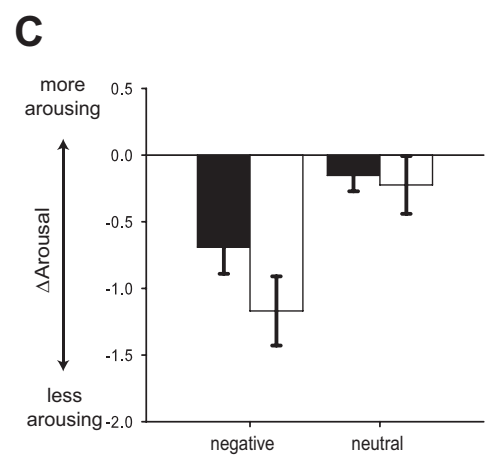

D

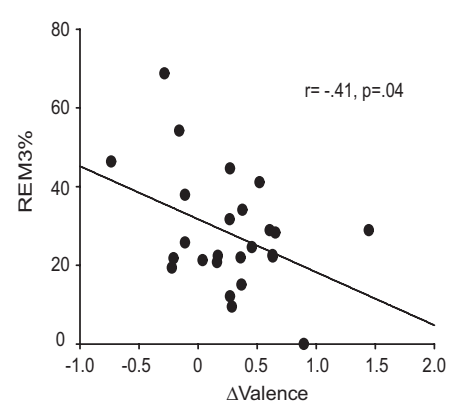

Figure 2. Performance and sleep physiology measures. $\boldsymbol{A}$, Hit rate in Session 2. $\boldsymbol{B}, \Delta$ Valence was computed as the SAM Valence ratings for Session 2 minus SAM valence ratings for Session 1. C, $\Delta$ Arousal was computed as the SAM Arousal rating for Session 2 minus SAM Arousal rating for Session 1 (error bars indicate SE). $D$, Relationship between REM and $\Delta$ Valence was significant. The percentage of the third quarter of the night spent in REM (REM3\%) negatively correlated with $\Delta$ Valence for negative items.
The ANOVA for $\Delta$ Valence revealed a significant Group $\times$ Valence interaction $\left(F_{(1,80)}=3.81, p=0.054\right) . \Delta$ Valence for negative pictures was higher in the Wake group (mean $=0.65$, SEM $=0.09$ ) compared with the Sleep group (mean $=0.39$, SEM $=0.07$ ), indicating that the initial negative ratings were attenuated more (i.e., more neutral) after a period of wake relative to sleep (Fig. 2 B). For neutral pictures, $\Delta$ Valence was similar 
across Sleep $($ mean $=-0.14, \mathrm{SEM}=0.04)$ and Wake $($ mean $=$ $-0.13, \mathrm{SEM}=0.06$ ) groups.

Likewise, arousal ratings for negative pictures were more attenuated over the $12 \mathrm{~h}$ break for the Wake group (mean $\Delta$ Arousal $=-1.17, \mathrm{SEM}=0.27$ ) compared with the Sleep group $($ mean $=-0.60$, SEM $=0.18) . \Delta$ Arousal for neutral pictures was reduced more over wake (mean $=-0.22, \mathrm{SEM}=0.21)$ than sleep $($ mean $=-0.15, \mathrm{SEM}=0.12$; Fig. $2 C$ ). However, the main effect of group was not significant $\left(F_{(1,80)}=1.51, p=0.22\right)$ nor was the Group $\times$ Valence interaction $\left(F_{(1,80)}=1.29, p=0.25\right)$.

Given that initial emotionality for targets and foils was similar (based on a preliminary study), we compared Session 2 ratings for targets (old stimuli) and foils (new stimuli), a measure of sleepdependent emotional processing used previously (Wagner et al., 2002). Specifically, the mean rating of foils was subtracted from the mean rating of targets in each valence category. The target - foil difference for mean valence did not differ for Sleep and Wake groups $\left(F_{(1,80)}=0.22, p=0.64\right)$ nor was there a Group $\times$ Valence interaction $\left(F_{(1,80)}=0.16, p=0.69\right)$. Similarly, the target - foil difference in mean arousal ratings did not differ for the Sleep and Wake groups (main effect of Group: $F_{(1,80)}=2.05, p=0.16$; Group $\times$ Valence interaction: $\left.F_{(1,80)}=0.34, p=0.56\right)$. This may suggest some level of generalization of the emotional response for targets to foils. However, caution must be taken in interpreting this response. While normative data were used to balance targets and foils a priori, by basing our analyses on subjective categorization, targets and foils may differ in subjective emotionality for the experimental groups and targets and foils were not balanced for number of items. Moreover, old items are being rated for the second time at test, and may innately differ from new items that are rated for the first time at test.

\section{Relationship between Memory and Valence}

Of particular interest in the present study was whether changes in memory are associated with changes in emotional reactivity over sleep. $\Delta$ Valence did not significantly correlate with hit rate for negative pictures (Wake: $r=0.25, p=0.19$; Sleep: $r=-0.096$, $p=0.49$ ). Likewise, for neutral pictures, the correlations between $\Delta$ Valence and hit rate were not significant for either the Sleep $(r=0.07, p=0.62)$ or Wake $(r=0.09, p=0.65)$ groups. $\Delta$ Arousal was also not a significant predictor of hit rate for negative (Sleep: $r=-0.17, p=0.22$; Wake: $r=-0.19, p=0.32$ ) or neutral (Sleep: $r=-0.15, p=0.27$; Wake: $r=0.06, p=0.78$ ) pictures. Likewise, when $d^{\prime}$ is used as a measure of memory, all correlations are nonsignificant.

\section{Associations between behavior and sleep physiology}

Late-night REM has been hypothesized to be critical to emotional memory consolidation (Wagner et al., 2001). Thus, we focused our comparisons on behavioral measures of negative pictures and measures of REM sleep (Table 3). We found no significant relationship between REM sleep time and hit rate for negative pictures $(r=-0.09, p=0.67), \Delta$ Valence $(r=-0.15, p=0.48)$, or $\Delta$ Arousal $(r=0.06, p=0.78)$.

Given that Wagner et al. (2001) measured REM in a 3 h sleep opportunity late in the night, with participants achieving $30 \mathrm{~min}$ of REM sleep, we focused further on REM sleep in the third quarter of the night (REM3\%) with the justification that this may be most similar to the 30 min obtained by Wagner et al.'s (2001) participants. Supporting this, our participants averaged 30.78 min of REM in the third quarter. We also separately considered REM4\%, as REM is thought to be richer as the night progresses (Mallick et al., 2011). REM3\% correlated significantly with
Table 3. Correlations between REM sleep measures and memory, $\Delta$ Valence, and $\Delta$ Arousal for negative pictures

\begin{tabular}{lrcc}
\hline & Hit rate & $\Delta$ Valence & $\Delta$ Arousal \\
\hline Total REM\% & & & \\
$r$ & -0.09 & -0.15 & 0.06 \\
$p$ & 0.67 & 0.48 & 0.78 \\
REM3\% & & & \\
$r$ & -0.23 & $-0.41^{*}$ & 0.22 \\
$p$ & 0.27 & 0.04 & 0.29 \\
REM4\% & & & \\
$r$ & 0.09 & -0.08 & 0.01 \\
$p$ & 0.65 & 0.69 & 0.97 \\
Total SWS\% & & & \\
$r$ & -0.26 & -0.02 & 0.04 \\
$p$ & 0.21 & 0.92 & 0.86 \\
SWS1\% & & & \\
$r$ & 0.02 & -0.02 & 0.18 \\
$p$ & 0.93 & 0.94 & 0.40 \\
SWS2\% & & & \\
$r$ & -0.17 & 0.04 & 0.05 \\
$p$ & 0.41 & 0.84 & 0.82 \\
\hline
\end{tabular}

Total REM\% = percentage of the night in rapid eye movement sleep; REM $3 \%$ = percentage of the third quarter of the night in REM; REM $4 \%$ = percentage of the fourth quarter of the night in REM; Total SWS $\%=$ percentage of the night in slow wave sleep; SWS1\% = percentage of the first quarter of the night in SWS; SWS2\% = percentage of the second quarter of the night in SWS. ${ }^{*} p<0.05$, two-tailed Pearson correlation.

$\Delta$ Valence; notably, contrary to predictions (Walker, 2009), this relationship was negative $(r=-0.41, p=0.04)$. That is, those participants with more REM $3 \%$ had less attenuation of negative reactivity (Fig. $2 D$ ). $\Delta$ Arousal did not correlate significantly with any of the REM measures (all ps $>0.28$ ). Likewise, none of the correlations between REM4\% and behavioral measures were significant (Table 3). Given that REM latency has also been shown to predict emotional memory processing (Nishida et al., 2009), we examined whether this measure (time to enter REM), predicted behavioral measures. We found no significant correlations between REM latency and hit rate $(r=0.17, p=0.43), \Delta$ Valence $(r=0.22, p=0.28)$, or $\Delta$ Arousal $(r=-0.29, p=0.16)$. Finally, a multiple regression analysis showed that $\Delta$ Valence, $\Delta$ Arousal, and REM (REM3\%, REM4\%, REM latency) were not significant predictors of hit rate for negative pictures ( $p s>0.15$ for all coefficients; adjusted $R^{2}=0.06$, n.s.).

Given the role of SWS on memory consolidation, we investigated the relationship of SWS and behavioral measures separately for negative and neutral pictures. Neither total SWS time nor SWS1\% reliably predicted hit rate, $\Delta$ Valence, or $\Delta$ Arousal (all ps $>0.21$; Table 3). Likewise, SWS2\% did not significantly correlate with behavioral measures with the exception of hit rate for neutral pictures where this correlation was, notably, negative ( $r=$ $-0.44, p=0.02$ ).

Finally, we probed the relationship between NREM Stage 2 and behavioral measures. NREM Stage 2 contains sleep spindles that are thought to underlie neural plasticity (Andrade et al., 2011). NREM Stage 2 in the last two quarters of the night did not correlate with any behavioral measures nor were significant correlations observed between behavior and total NREM Stage 2 (all ps $>0.46)$. All sleep-stage correlations were in the same direction and nonsignificant when $d^{\prime}$ was used.

\section{Circadian effects}

The Morning and Evening groups were included to examine whether differences observed between the Sleep and Wake groups reflect circadian influences on cognition. A comparison of valence and arousal ratings in the Encoding Phase failed to sup- 
port a circadian account: the Morning and Evening groups rated Valence (negative: $F_{(1,22)}=0.19, p=0.66$; neutral: $F_{(1,22)}=0.06$, $p=0.81$ ) and Arousal (negative: $F_{(1,22)}=1.58, p=0.22$; neutral: $\left.F_{(1,22)}=0.14, p=0.71\right)$ similarly.

A mixed ANOVA for hit rate revealed that the main effect of Group (Morning vs Evening) was not significant $\left(F_{(1,22)}=2.69\right.$, $p=0.12)$. The Valence $\times$ Group interaction was also not significant $\left(F_{(1,22)}=0.22, p=0.64\right)$. However, there was a main effect of Valence $\left(F_{(1,22)}=4.07, p=0.04\right)$. Negative pictures were remembered better than neutral pictures in the $12 \mathrm{~h}$ groups as well.

Similarly, ANOVA for recognition memory discriminability $\left(d^{\prime}\right)$ revealed no significant main effect of group $\left(F_{(1,22)}=\right.$ 2.73, $p=0.12)$ and no Group $\times$ Valence interaction $\left(F_{(1,22)}=\right.$ $0.24, p=0.63)$. There was also no main effect of Valence in $d^{\prime}$ measures $\left(F_{(1,22)}=0.89, p=0.36\right)$. ANOVA for response bias (c) revealed no significant main effect of $\operatorname{Group}\left(F_{(1,22)}=1.31\right.$, $p=0.27)$, no main effect of Valence $\left(F_{(1,22)}=1.71, p=0.21\right)$, and no significant Group $\times$ Valence interaction $\left(F_{(1,22)}=\right.$ 3.42, $p=0.08)$.

A mixed ANOVA for $\triangle$ Valence revealed no significant effect of Group (Morning vs Evening; $F_{(1,22)}=0.30, p=0.60$ ). $\Delta$ Valence for negative pictures was significantly different from zero in both groups (Evening: $t_{(11)}=4.82, p=0.001$; Morning: $\left.t_{(11)}=6.44, p<0.001\right)$. Thus, even a $45 \mathrm{~min}$ interval was enough to reduce the negativity of the perceived valence (i.e., items are rated as less negative). Interestingly, $\Delta$ Valence over $45 \mathrm{~min}$ awake (Morning and Evening combined) was similar to the $\Delta$ Valence observed following $12 \mathrm{~h}$ awake $\left(F_{(1,50)}=0.64, p=0.42\right)$ and was not significantly different from $\Delta$ Valence for the $12 \mathrm{~h}$ Sleep group $\left(F_{(1,76)}=1.98, p=0.16\right)$.

$\Delta$ Arousal ratings also did not differ for the Morning and Evening groups $\left(F_{(1,20)}=0.41, p=0.53\right)$. For both Morning and Evening groups, $\Delta$ Arousal for negative pictures was not different from zero (Evening: $t_{(11)}=-0.56, p=0.59$; Morning: $t_{(11)}=$ $0.73, p=0.48)$. Although $\Delta$ Arousal did not change within 45 min, following $12 \mathrm{~h}$ of wake, $\Delta$ Arousal was greatly reduced (45 min groups vs Wake group; $\left.F_{(1,50)}=10.9, p=0.002\right)$. $\Delta$ Arousal following $45 \mathrm{~min}$ wake was not significantly different from $\Delta$ Arousal following $12 \mathrm{~h}$ sleep $\left(F_{(1,76)}=3.25, p=0.08\right)$. Together, these results suggest a differential effect of time on valence and arousal.

\section{Discussion}

While numerous recent studies have demonstrated sleepdependent consolidation of emotional memories, here we provide the first examination of how memory processing relates to changes in emotional reactivity over sleep. Recognition memory was better following sleep compared with wake. Additionally, a period of wake was associated with attenuation of negative ratings whereas a period of sleep was associated with relative maintenance of the initial negative ratings. Therefore sleep may, in fact, be protective of the emotional salience of a stimulus just as sleep protects the emotional memory.

Walker (2009) suggested a reciprocal relationship between emotional memory consolidation and emotional reactivity maintenance, positing that as negative memories are consolidated, the emotional reactivity is attenuated. Importantly, we fail to find evidence of such a relationship. Instead, our results suggest that sleep's effect on memory and emotion may be independent. First, if the processes protecting emotional reactivity over sleep overlap at all with those processes underlying sleepdependent consolidation of the emotional memory, individual differences in these measures should correlate (for a discussion of individual difference approach, see Zelaznik et al., 2002). However, hit rate and the change in emotional reactivity ( $\Delta$ Valence or $\Delta$ Arousal) following sleep were not related. Second, while we find evidence that protection of emotional salience is REMdependent, no such relationship exists for memory and measures of REM (quantity or latency). This latter result is inconsistent with the work of Nishida et al. (2009), who found a correlation between REM within a brief nap and a measure of $d^{\prime}$ that included both presleep and postsleep learning. Lacking a comparable measure of memory, one can only speculate as to whether these results are indeed conflicting. Together, our results may suggest independent processing of memory and emotional reactivity over sleep. A caveat on this interpretation is the reliance on null results in correlational measures. Nonetheless, what is clear is that the association between memory and changes in emotional reactivity is not reciprocal as previously suggested.

In contrast to previous studies (Wagner et al., 2001; Payne et al., 2008), sleep did not preferentially act on memory for emotionally negative items over neutral items. This difference is not likely to be attributable to the incidental nature of the task: Although intentional memories are subject to greater memory benefits from sleep, emotion is thought to add intent to incidental encoding (Wilhelm et al., 2011). Rather, the lack of preferential consolidation for emotional memories may be due to differences in experimental paradigms across this literature. Studies vary in stimuli content (e.g., faces, scenes), the array of emotional arousal and valence, and presentation times. Given the myriad of paradigms used across this literature, direct comparisons may not be justified. However, it should be noted that Hu et al. (2006) also failed to find a preferential benefit for arousing stimuli relative to neutral stimuli for Remember judgments; this preferential benefit was only seen for Know judgments. Moreover, our results are consistent with two recent studies that also failed to find preferential benefits of sleep for emotionally negative relative to neutral stimuli (Campanella and Hamann, 2011; Lewis et al., 2011). Collectively, the conditions underlying preferential consolidation of emotional memories over sleep require closer examination.

We considered whether differences for the Sleep and Wake groups reflect circadian influences on performance. For this reason, we included two control groups: the Morning and Evening groups. If, for instance, memory recall peaks when cortisol levels are highest, we would expect the Morning group to have more accurate recall than the Evening group. Such was not the case. We found no differences in hit rate (or $\Delta$ Valence, $\Delta$ Arousal, or emotional ratings) for these groups. In fact, mean accuracy was greater for the Evening group than the Morning group, although not significant. However, circadian influences may have been greatest on memory encoding rather than retrieval. This would explain the slightly greater performance of the Evening relative to the Morning group and likewise for the Sleep relative to the Wake group. This explanation is unlikely for three reasons. First, a circadian influence on encoding could only explain the sleep benefit on negative items. The memory for neutral items was superior for the Sleep relative to the Wake group and yet the memory for neutral items was nearly identical for Morning and Evening groups. Second, the association between $\Delta$ Valence and REM supports an active role of sleep, at least with respect to the modulation of the emotional response. Finally, previous work has ruled out circadian influences on emotional memory consolidation 
(Payne and Kensinger, 2011). Thus, we favor the interpretation of sleep playing an active role in emotional memory consolidation and modulation of emotional reactivity.

Emotional reactivity, which was attenuated over wake, was less attenuated over sleep. That is, a picture that was initially deemed highly negative was rated as substantially less negative following $12 \mathrm{~h}$ awake but only mildly less negative following sleep. One possibility is that such changes reflect habituation. However, one might expect that sleep would enhance habituation. By repeatedly reactivating the memory, as posited to occur during sleep (Wilson and McNaughton, 1994), one might become habituated to the emotional response provoked by the stimulus. Alternatively, such changes may reflect encoding and consolidation of a habituation memory (Pace-Schott et al., 2011a). Neuroplastic changes brought about by viewing negative stimuli can be viewed as an early stage in habituation subserved, perhaps, by plasticity in emotion-related structures (e.g., basolateral amygdala). Such neuroplastic changes could be consolidated over sleep, resulting in a greater emotional response in the Recognition Phase. Recent neuroimaging studies reveal increased amygdala activation and increased connectivity between the amygdala and the limbic system for retrieval of negative stimuli following sleep compared with wake (Lewis et al., 2011; Payne and Kensinger, 2011). It could be speculated that increased activation and connectivity are markers of such neuroplastic changes that enhance consolidation and maintain emotional reactivity.

Overall, sleep-dependent changes in emotional reactivity were similar for measures of valence and arousal. Although functional neuroimaging studies initially suggested that the brain bases for the memory enhancing effects of valence and arousal were discrete, it is now known that neural systems supporting these two dimensions can interact to enhance memory (Kensinger, 2004; LaBar and Cabeza, 2006; Pierce and Kensinger, 2011). More specifically, whereas arousal-related memory enhancement was originally thought to be associated with amygdala connectivity while valence-related enhancement was associated with interactions between medial temporal and prefrontal cortices (Kensinger, 2004; Kensinger and Corkin, 2004; LaBar and Cabeza, 2006), recent studies suggest that these two neural systems can interact to promote memory (Mickley Steinmetz et al., 2010; Waring and Kensinger, 2011). However, although the net effects of sleep were similar for valence and arousal, the time course of these changes differed. While changes in valence occurred early (within $45 \mathrm{~min}$ ), changes in arousal occurred later, with no changes observed following $45 \mathrm{~min}$ of wake. This result is consistent with other work illustrating that the memory-enhancing effects of arousing pictures over neutral pictures also changes over time (LaBar and Cabeza, 2006).

In summary, with an incidental recognition memory task using picture stimuli, we demonstrate that a period of sleep is associated with better memory and less attenuation of reactivity for negative pictures. Although attenuation of emotional reactivity to a briefly encountered, novel stimulus such as an IAPS picture may be adaptive from a point of view that emphasizes the potential mental health benefit of such a mechanism, an evolutionary perspective might emphasize the exact opposite effect. That is, maintenance of salience might have been of survival value to ancestral humans. By maintaining the negative tone of the strengthened memory trace, the individual will not only remember the emotional event but also the degree of threat associated with it.

\section{Notes}

Supplemental material for this article is available at http://cognaclab. com/wp/wp-content/uploads/EmoSOM.pdf. This material has not been peer reviewed.

\section{References}

Andrade KC, Spoormaker VI, Dresler M, Wehrle R, Holsboer F, Sämann PG, Czisch M (2011) Sleep spindles and hippocampal functional connectivity in human NREM sleep. J Neurosci 31:10331-10339.

Bradley MM, Lang PJ (2007) The international affective picture system (IAPS) in the study of emotion and attention. In: Handbook of emotion elicitation and assessment (JA Coan, JJB Allen, eds) pp 29-46. NY: Oxford UP.

Buysse DJ, Reynolds CF 3rd, Monk TH, Berman SR, Kupfer DJ (1989) The Pittsburgh Sleep Quality Index: a new instrument for psychiatric practice and research. Psychiatry Res 28:193-213.

Campanella C, Hamann S (2011) Examining the effects of post-learning sleep on consolidation of associative emotional declarative memories. Paper presented at 2011 Annual Meeting of Cognitive Neuroscience Society, San Francisco, April.

Carskadon MA, Dement WC, Mitler MM, Guilleminault C, Zarcone VP, Spiegel R (1976) Self-reports versus sleep laboratory findings in 122 drug-free subjects with complaints of chronic insomnia. Am J Psychiatry 133:1382-1388.

Diekelmann S, Born J (2010) The memory function of sleep. Nat Rev Neurosci 11:114-126.

Gais S, Born J (2004) Declarative memory consolidation: mechanisms acting during human sleep. Learn Mem 11:679-685.

Hu P, Stylos-Allan M, Walker MP (2006) Sleep facilitates consolidation of emotional declarative memory. Psychol Sci 17:891-898.

Iber C, Ancoli-Israel S, Chesson A, Quan SF (2007) The AASM manual for the scoring of sleep and associated events: rules, terminology, and technical specification, 1st ed. Westchester, IL: American Academy of Sleep Medicine.

Kensinger EA (2004) Remembering emotional experiences: the contribution of valence and arousal. Rev Neurosci 15:241-251.

Kensinger EA, Corkin S (2004) Two routes to emotional memory: distinct neural processes for valence and arousal. Proc Natl Acad Sci U S A 101:3310-3315.

Killgore WD, Balkin TJ, Wesensten NJ (2006) Impaired decision making following $49 \mathrm{~h}$ of sleep deprivation. J Sleep Res 15:7-13.

LaBar KS, Cabeza R (2006) Cognitive neuroscience of emotional memory. Nat Rev Neurosci 7:54-64.

Lang PJ (1980) Behavioral treatment and bio-behavioral assessment: computer applications. In: Technology in mental health care delivery systems (JB Sidowski, JH Johnson, TA Williams, eds), pp119-137. Norwood, NJ: Ablex.

Lang PJ, Bradley MM, Cuthbert BN (2005) International affective picture system (IAPS): affective ratings of pictures and instruction manual. Technical Report A-6. Gainesville, FL: University of Florida.

Lewis PA, Cairney S, Manning L, Critchley HD (2011) The impact of overnight consolidation upon memory for emotional and neutral encoding contexts. Neuropsychologia 49:2619-2629.

Mallick BN, Pandi-Permual SR, McCarley RW, Morrison AR, eds (2011) Rapid eye movement sleep: regulation and function. Cambridge: Cambridge UP.

Marshall L, Born J (2007) The contribution of sleep to hippocampusdependent memory consolidation. Trends Cogn Sci 11:442-450.

Marshall L, Helgadóttir H, Mölle M, Born J (2006) Boosting slow oscillations during sleep potentiates memory. Nature 444:610-613.

Macmillan NA, Creelman CD (1990) Response bias: characteristics of detection theory, threshold theory and "nonparametric" indexes. Psych Bull 107:401-413.

Mickley Steinmetz KR, Addis DR, Kensinger EA (2010) The effect of arousal on the emotional memory network depends on valence. Neuroimage 53:318-324.

Nishida M, Pearsall J, Buckner RL, Walker MP (2009) REM sleep, prefrontal theta, and the consolidation of human emotional memory. Cereb Cortex 19:1158-1166.

Pace-Schott EF, Milad MR, Orr SP, Rauch SL, Stickgold R, Pitman RK (2009) Sleep promotes generalization of extinction of conditioned fear. Sleep $32: 19-26$ 
Pace-Schott EF, Shepherd E, Spencer RM, Marcello M, Tucker M, Propper RE, Stickgold R (2011a) Napping promotes inter-session habituation to emotional stimuli. Neurobiol Learn Mem 95:24-36.

Pace-Schott EF, Nave G, Morgan A, Spencer RM (2011b) Sleep-dependent modulation of affectively guided decision-making. J Sleep Res. Advance online publication. doi:10.1111/j.1365-2869.2011.00921.x.

Payne JD, Kensinger EA (2011) Sleep leads to changes in the emotional memory trace: evidence from FMRI. J Cogn Neurosci 23:1285-1297.

Payne JD, Stickgold R, Swanberg K, Kensinger EA (2008) Sleep preferentially enhances memory for emotional components of scenes. Psychol Sci 19:781-788.

Pierce BH, Kensinger EA (2011) Effects of emotion on associative recognition: valence and retention interval matter. Emotion 11:139-144.

Riemann D (2007) Insomnia and comorbid psychiatric disorders. Sleep Med 8 [Suppl 4]:S15-S20.

Schabus M, Gruber G, Parapatics S, Sauter C, Klösch G, Anderer P, Klimesch W, Saletu B, Zeitlhofer J (2004) Sleep spindles and their significance for declarative memory consolidation. Sleep 27:1479-1485.

Silva GE, Goodwin JL, Sherrill DL, Arnold JL, Bootzin RR, Smith T, Walsleben JA, Baldwin CM, Quan SF (2007) Relationship between reported and measured sleep times: the Sleep Heart Health Study (SHHS). J Clin Sleep Med 3:622-630.

Stickgold R (2005) Sleep-dependent memory consolidation. Nature 437: 1272-1278.

St. Jacques PL, Dolcos F, Cabeza R (2009) Effects of aging on functional connectivity of the amygdala for subsequent memory of negative pictures: a network analysis of functional magnetic resonance imaging data. Psychol Sci 20:74-84.

Wagner U, Gais S, Born J (2001) Emotional memory formation is enhanced across sleep intervals with high amounts of rapid eye movement sleep. Learn Mem 8:112-119.

Wagner U, Fischer S, Born J (2002) Changes in emotional responses to aversive pictures across periods rich in slow-wave sleep versus rapid eye movement sleep. Psychosom Med 64:627-634.

Wagner U, Hallschmid M, Rasch B, Born J (2006) Brief sleep after learning keeps emotional memories alive for years. Biol Psychiatry 60:788-790.

Walker MP (2009) The role of sleep in cognition and emotion. Ann N Y Acad Sci 1156:168-197.

Walker MP, Stickgold R (2006) Sleep, memory, and plasticity. Annu Rev Psychol 57:139-166.

Walker MP, van der Helm E (2009) Overnight therapy? The role of sleep in emotional brain processing. Psychol Bull 135:731-748.

Waring JD, Kensinger EA (2011) How emotion leads to selective memory: neuroimaging evidence. Neuropsychologia 49:1831-1842.

Wilhelm I, Diekelmann S, Molzow I, Ayoub A, Mölle M, Born J (2011) Sleep selectively enhances memory expected to be of future relevance. J Neurosci 31:1563-1569.

Wilson MA, McNaughton BL (1994) Reactivation of hippocampal ensemble memories during sleep. Science 265:676-679.

Zelaznik HN, Spencer RM, Ivry RB (2002) Dissociation of explicit and implicit timing in repetitive tapping and drawing movements. J Exp Psychol Hum Percept Perform 28:575-588. 